\title{
Photocurrents at polarized liquid|liquid interfaces enhanced by a gold nanoparticle film $\dagger$
}

\author{
Delphine Schaming, ${ }^{a}$ Mohamad Hojeij, ${ }^{a}$ Nathalie Younan, ${ }^{a}$ Hirohisa Nagatani, ${ }^{a b}$ \\ Hye Jin Lee ${ }^{c}$ and Hubert H. Girault*a
}

Received 24th June 2011, Accepted 17th August 2011

DOI: $10.1039 / \mathrm{c1cp22072a}$

Photocurrent responses associated with the interfacial quenching of the photo-excited water-soluble zinc meso-tetra(4-carboxyphenyl)porphyrin (ZnTPPC) by ferrocene have been studied at a water|1,2-dichloroethane interface in the absence and in the presence of adsorbed gold nanoparticles. Upon addition of methanol, a mirror-like gold film is formed and an important enhancement of the photocurrent response can be observed. Intensity modulated photocurrent spectroscopy experiments (IMPS) have been performed, in order to deconvolute in the frequency domain the contribution from the competition between the recombination and the product separation arising after the electron transfer, and the attenuation associated with the resistance and interfacial capacitance $\left(R C_{\text {int }}\right)$ time constant of the cell.

\section{Introduction}

With the goal of solar energy conversion, many studies have shown how dye-sensitized semiconducting materials can photogenerate and transport charge carriers. However, interfaces involving solid-state components can be subject to structural defects, which can act as traps or recombination centers for charge carriers, and consequently can lead to energy losses. The use of soft molecular interfaces may circumvent this problem, and dye-sensitized, polarized Interfaces between Two Immiscible Electrolyte Solutions (ITIES) provide in this respect an interesting approach. For these defect-free interfaces, the separation of sensitizers and quenchers by a liquid/liquid junction is easily achieved and heterogeneous photoinduced electron transfer reactions can be investigated by measuring photocurrents.

In previous works, we have already described photocurrent responses arising from the heterogeneous quenching of watersoluble zinc porphyrins at the water|1,2-dichloroethane (DCE) interface. ${ }^{1-6}$ These responses rely on the distinctive adsorption properties of the porphyrins at the interface. For instance, the adsorption of the zinc meso-tetrakis(4-carboxyphenyl)porphyrin

${ }^{a}$ Laboratoire d'Electrochimie Physique et Analytique,

Ecole Polytechnique Fédérale de Lausanne, Station 6,

CH-1015 Lausanne, Switzerland.E-mail: hubert.girault@epfl.ch;

Fax: + 412169336 67; Tel: + 41216933145

${ }^{b}$ Faculty of Chemistry, Institute of Science and Engineering,

Kanazawa University, Kakuma, Kanazawa 920-1192, Japan

${ }^{c}$ Department of Chemistry, Kyungpook National University,

1370 Sankyuk-dong, Buk-gu, Daegu-city, 702-701, Republic of Korea

$\dagger$ Electronic supplementary information (ESI) available: UV-visible

and electrochemical studies of ZnTPPC and explanations about the

IMPS theory. See DOI: 10.1039/c1cp22072a
(ZnTPPC) has been studied by Surface Second Harmonic Generation (SSHG) to monitor its surface concentration and aggregation, and the photocurrent responses with different quenchers were shown to occur when the porphyrins are not aggregated. This feature is rather different from the one obtained for other water-soluble porphyrins such as the zinc meso-tetrakis(4-sulfonatophenyl)porphyrin (ZnTPPS) or the zinc meso-tetrakis $(N$-methyl-4-pyridyl)porphyrin (ZnTMPyP) for which the individual photoresponses are negligible. Nevertheless, these two last porphyrins are able to form selfassembled ion-pair structures at the interface with particular interfacial solvation properties that give rise to high photocurrent responses.

In the last decade, many studies have investigated the effect of plasmonics to improve photovoltaic devices. Indeed, plasmonics can be used to improve absorption resulting in a considerable reduction in the physical thickness of solar photovoltaic absorber layers, and yielding new options for solar-cell design. ${ }^{7}$ For instance, metallic nanoparticles can be used as antennas in which the plasmonic near-field is coupled to the semiconductor, increasing its effective absorption crosssection. It is now well established that upon excitation the plasmon resonance of metallic nanoparticles leads to the formation of a strong local electric field around the nanoparticles. This strong electric field can further enhance the electronic excitation of fluorophores present close to the metallic nanoparticles. Several examples of this concept have recently appeared that demonstrate enhanced photocurrents owing to the plasmonic near-field coupling. ${ }^{8-14}$

For example, Akiyama et al. have recently studied photocurrent generation of porphyrins adsorbed onto ITO electrodes modified by gold films, and have observed an enhancement of 
the photocurrent responses due to the effect of localized surface plasmon resonance appearing at the surface of gold films compared to the porphyrins used without these gold nanostructures. ${ }^{15-17}$

Surface plasmon resonance (SPR) can also occur at liquid|liquid interfaces in the presence of metallic films formed by the assembly of nanoparticles, but it is rather difficult to distinguish localized SPR (LSPR) effects generated by adsorbed nanoparticles from SPR propagating along the metallic film. In 2007, Cohanoschi et al. have shown the enhancement of interfacial fluorescence of dyes at the water|xylene interface in the presence of a film of gold nanoparticles. ${ }^{18}$ In 2010, we have studied the angular dependence of the interfacial reflectivity of interfacial gold films in a pseudo-Kretschmann configuration showing that propagating SPR can be observed at soft interfaces. ${ }^{19}$ Very recently, one of us has shown that the presence of gold nanoparticles in aqueous solutions can also enhance photocurrents at ITIES resulting from the quenching of ZnTPPC by ferrocene, and concluded that this enhancement resulted from LSPR. ${ }^{20}$ The aim of this paper is to study this effect in the presence of a gold film showing propagating properties.

Formation of metallic films at liquid|liquid interfaces from colloidal solutions of metallic nanoparticles has been known for many years since the pioneering work of Yogev and Efrima using different recipes, ${ }^{21}$ but the mechanisms for the film formation remain often elusive. For example, a practical way to obtain a gold film consists in adding alcohol (methanol or ethanol usually) at the interface between an aqueous solution of gold nanoparticles and an organic phase, but the rational of this approach is still not clear. ${ }^{15,16,18,19,22,23}$

We report in this work the enhancement of photocurrent responses of the water-soluble zinc porphyrin ZnTPPC by the plasmon resonance effect of a mirror-like gold film at the water|DCE interface. Kinetic information about the chemical processes involved in these photocurrent responses has been investigated by using intensity modulated photocurrent spectroscopy (IMPS).

\section{Experimental section}

\section{Chemicals}

All electrolyte solutions were prepared from analytical grade reagents, which were purchased commercially and used without further purification.

The dissolution of ZnTPPC (purchased from Frontier Scientific) was performed in an aqueous solution of $0.1 \mathrm{M}$ of $\mathrm{NaOH}$, and afterwards, the $\mathrm{pH}$ of the solution was adjusted to 5.8 by addition of $1 \mathrm{M}$ aqueous solution of $\mathrm{HCl}$.

The supporting electrolyte used in the organic phase was bis(triphenylphosphoranylidene)ammonium tetrakis(pentafluorophenyl)borate (BATB). This electrolyte was prepared by metathesis of bis(triphenylphosphoranylidene)ammonium chloride $(\mathrm{BACl})$ and lithium tetrakis(pentafluorophenyl)borate ethyl etherate (LiTB) in a $2: 1$ mixture of methanol and water, followed by recrystallisation in acetone.

Gold nanoparticles (gold NPs) were synthesized by the Turkevich method. ${ }^{24}$ For that, $95 \mathrm{~mL}$ of an aqueous solution of $0.27 \mathrm{mM}$ of $\mathrm{HAuCl}_{4}$ were heated to the boiling point, and then $5 \mathrm{~mL}$ of $1 \%$ sodium citrate solution were added with

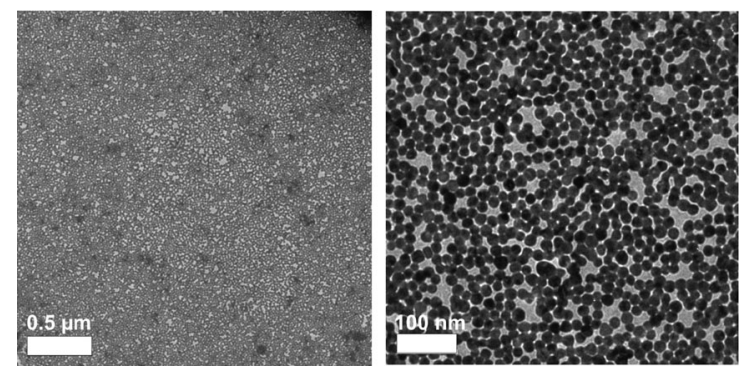

Fig. 1 TEM micrographs of the gold NP film at different resolutions.

vigorous stirring. The solution was heated for $1 \mathrm{~h}$, and then the obtained red solution was left for cooling. As prepared, the concentration of the gold NPs solution is around $2 \mathrm{nM}$. The characterization of the gold NPs was performed by transmission electron microscopy (TEM) using a Philips CM20. The NPs are found to be spherical with an average size of $16 \pm 2 \mathrm{~nm}$. By measuring over 100 particles with TEM, the full width at half maximum (FWHM) of the size distribution peak of the particles is found to be less than $15 \%$ of the median size.

A TEM picture is depicted in Fig. S1. $\dagger$

To obtain the mirror-like gold film at the liquid|liquid interface, $10 \mathrm{~mL}$ of three times diluted previous solution of gold NPs $(0.7 \mathrm{mM})$ were placed above $15 \mathrm{~mL}$ of DCE. Then, $5 \mathrm{~mL}$ of methanol were vigorously injected at the interface followed by stirring for $10 \mathrm{~min}$. After that, the DCE/water interface was slowly re-formed, and a thin film with blue and yellow reflectances appeared at the interface. TEM micrographs of the film are depicted in Fig. 1 at different resolutions. In order to obtain these TEM micrographs, a part of the film was carefully "scooped" to the copper grids by slowly sliding off the films. The micrographs show a homogeneous monolayer compact film with a thickness of almost $16 \mathrm{~nm}$ (average size of the NPs). This is in agreement with already published works on such metallic NP films. ${ }^{25,26}$

\section{Photocurrent setup and IMPS experiments}

A four-electrode system using an Autolab PGSTAT30 potentiostat was used to carry out electrochemical and photocurrent studies. Experiments were performed in an optical glass cell $(25 \times 40 \times$ $55 \mathrm{~mm}$ ) capped by a Teflon top including two capillaries for the reference electrode and the counter electrode of the organic phase. Two platinum grids were used as counter electrodes for the two phases, and two $\mathrm{Ag} \mid \mathrm{AgCl}$ electrodes were used as reference electrodes. The Luggin capillary used for the organic phase reference electrode was filled by an aqueous solution containing $1 \mathrm{mM}$ of $\mathrm{BACl}$ and $10 \mathrm{mM}$ of $\mathrm{LiCl}$, in order to ensure the electrical connection. All Galvani potential differences across the water|DCE interface were estimated by taking the formal transfer potential of tetramethylammonium $\Delta_{\mathrm{o}}^{\mathrm{w}} \phi_{\mathrm{TMA}^{+}}^{0}$ as $0.160 \mathrm{~V} .^{27}$

Photocurrent studies were carried out with a $\mathrm{He}-\mathrm{Cd}$ laser at $442 \mathrm{~nm}$ (Omnichrome Series 74). The interface was illuminated in total reflection mode from the organic phase (with an incident angle of about $80^{\circ}$ (with respect to the surface normal), unless otherwise indicated). A mechanical shutter was used to control the light on and off. The cross section of the spot beam with the interface was $c a .5 \mathrm{~mm}$ in diameter, and the photocurrent density was calculated using this spot area. 


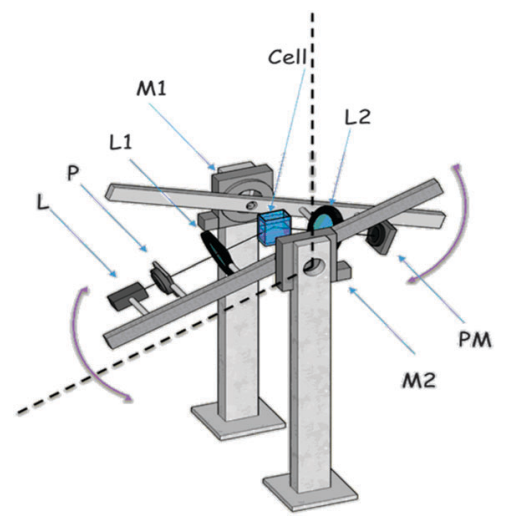

Scheme 1 Custom-built setup for surface plasmon resonance at the liquid|liquid interface.

IMPS experiments were performed with a lock-in amplifier SR830 DSP from Stanford Research Systems to record the phase difference between the shutter and the photocurrent response (i.e. the real and the imaginary parts of the photocurrent responses).

\section{SPR setup}

Scheme 1 shows the custom-built experimental setup used for the surface plasmon resonance (SPR) experiment at the liquid|liquid interface. A $5 \mathrm{~mW}$ Intelite laser at $532 \mathrm{~nm}$ was used as the excitation source. A p-polarizer (Newport) was used to favour surface plasmon resonance. A lens L1 was used to focus the beam at the center of the meniscus of the interface. Another lens L2 was used to collect the reflected beam into a sensitive power meter (Thorlabs PM100) used to detect the signal. Two motors from Thorlabs, M1 and M2, were used to provide a symmetrical rotation of the laser and the power meter around the optical glass cell, in order to change the angle of incidence. Using the Labview software, the reflected intensity was recorded as a function of the angle of incidence. The measurement was conducted in the dark.

In order to avoid light diffusion problems at small grazing angles, the cell was previously silanized by using dimethyldichlorosilane, in order to obtain a meniscus with a concavity in the upper aqueous phase, the silanized glass walls being preferentially wetted by the organic solvent.

The simulated SPR curves were obtained by using the program developed by the research group of Robert M. Corn at the University of California, Irvine, ${ }^{28}$ where the liquid|metallic film|liquid system was reduced to a three-layer model. The refractive indexes of gold films were adjusted to the wavelength referring to the experimental values of Johnson and Christy. ${ }^{29}$ The refractive indexes of DCE and of water were taken as 1.445 and 1.333 , respectively.

\section{Results and discussion}

\section{Electrochemical study}

Fig. 2 (full line) shows the cyclic voltammogram of the water|DCE interface in the dark containing ZnTPPC (without gold NPs) in the aqueous phase and ferrocene ( $\mathrm{Fc}$, used as a quencher in the following photocurrent experiments) in the organic phase. The employed electrochemical cell is schematically displayed in Scheme 2.

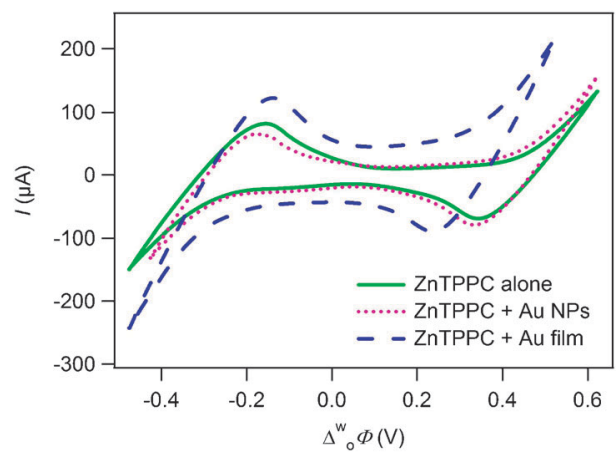

Fig. 2 Cyclic voltammograms recorded at $50 \mathrm{mV} \mathrm{s}^{-1}$ in the presence of $0.1 \mathrm{mM} \mathrm{ZnTPPC}$ and $1 \mathrm{mM} \mathrm{Fc}$ in the aqueous and DCE phases, respectively. Full line: without gold NPs; dotted line: with gold NPs added to the aqueous phase; dashed line: in the presence of the gold NP film formed by addition of methanol.

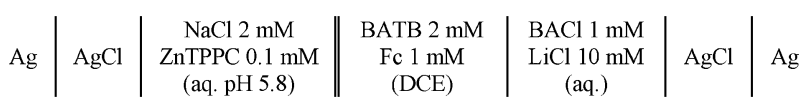

Scheme 2 Electrochemical cell in the absence of gold NPs.

By convention, a positive current corresponds to the transfer of a positive charge from the aqueous to the organic phase. The voltammogram exhibits a potential window of nearly $500 \mathrm{mV}$ in which no significant Faradaic response is observed in the dark. The positive potential limit is determined here by the transfer of $\mathrm{Na}^{+}$and $\mathrm{H}^{+}$from water to DCE, while the negative limit is given by the transfer of $\mathrm{Cl}^{-}$, also from water to DCE. The absence of any Faradaic response within these limits indicates that no electron transfer occurs via the ground state of the porphyrin.

The large $i R$-drop observed in Fig. 2 is due to the cell design with a large surface area $\left(10 \mathrm{~cm}^{2}\right)$ well adapted to spectroscopic measurements but clearly not ideal for voltammetry.

When gold NPs are added to the aqueous phase (the concentration of porphyrin is maintained constant), no significant changes are observed (Fig. 2, dotted line).

When methanol is vigorously added to the interface, a film with blue and yellow reflectances appears at the interface, corresponding to a thin gold film. Indeed, when a water-miscible solvent with a lower dielectric constant, such as methanol, is added, the surface charge density of the nanoparticles decreases, most likely due to competitive adsorption of alcohol with citrate on the gold NPs, leading to their adsorption to the interface. ${ }^{2,25,30}$ Concerning the electrochemical properties of this gold NP film (Fig. 2, dashed line), a larger capacitive current is observed, indicating well the presence of an adsorbed layer at the interface. Moreover, the potential window changes. This can be explained by the modification of the solvent (addition of methanol), which leads to a variation of the values of the transfer potential of the different species across the interface.

\section{Photocurrent enhancement by plasmon resonance}

Photocurrent generation has been investigated by illumination at $442 \mathrm{~nm}$ (close to the Soret band, see Fig. S2 $\dagger$ ) in a total internal reflection mode, allowing the excitation of the porphyrin. 
In the presence of ZnTPPC alone in the aqueous phase, positive photocurrent transient responses are observed throughout the potential window (Fig. 3, full lines). The elementary steps involved in this heterogeneous quenching can be described as:

$$
\mathrm{ZnTPPC}_{\mathrm{w}} \stackrel{h \nu}{\longrightarrow} \mathrm{ZnTPPC}_{\mathrm{w}}^{*} \text { Photoexcitation }
$$

$$
\mathrm{ZnTPPC}_{\mathrm{w}}^{*} \stackrel{k_{\mathrm{d}}}{\longrightarrow} \mathrm{ZnTPPC}_{\mathrm{w}} \text { Decay of the excited state }
$$

$\mathrm{ZnTPPC}_{\mathrm{w}}^{*}+\mathrm{Fc}_{\mathrm{o}} \stackrel{k_{\mathrm{et}}}{\longrightarrow}\left[\mathrm{ZnTPPC}^{\bullet-} \ldots \mathrm{Fc}^{+}\right]_{\text {int }}$ Electron transfer

$\left[\mathrm{ZnTPPC}^{\bullet-} \ldots \mathrm{Fc}^{+}\right]_{\mathrm{int}} \stackrel{k_{\mathrm{ps}}}{\longrightarrow} \mathrm{ZnTPPC}_{\mathrm{w}}^{\bullet-}+\mathrm{Fc}_{\mathrm{o}}^{+}$Products separation

$\left[\mathrm{ZnTPPC}^{\bullet-} \ldots \mathrm{Fc}^{+}\right]_{\text {int }} \stackrel{k_{\mathrm{rec}}}{\longrightarrow} \mathrm{ZnTPPC}_{\mathrm{w}}+\mathrm{Fc}_{\mathrm{o}} \quad$ Recombination

where the subindexes "w", "o" and "int" correspond to the aqueous, organic and interfacial regions, respectively, and
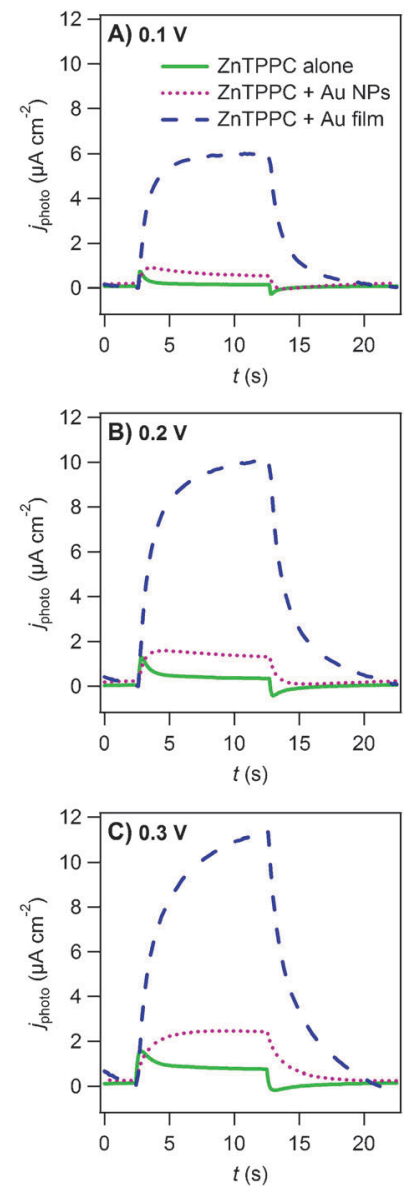

Fig. 3 Photocurrent transient measurements at (A) $0.1 \mathrm{~V}$, (B) $0.2 \mathrm{~V}$ and (C) $0.3 \mathrm{~V}$ obtained with the biphasic system containing $0.1 \mathrm{mM}$ ZnTPPC and $1 \mathrm{mM} \mathrm{Fc}$ in the aqueous and DCE phases, respectively. Full line: without gold NPs; dotted line: with gold NPs added to the aqueous phase; dashed line: in the presence of the gold NP film formed by addition of methanol. Illumination was provided at $442 \mathrm{~nm}$. $k$ the pseudo first order rate constants of the different involved processes.

At polarized interfaces, electron exchange reactions between $\mathrm{ZnTPPC}$ and $\mathrm{Fc}$ are associated with Faradaic responses, and by convention, the electron transfer step (eqn (3)) between the photoexcited porphyrin (ZnTPPC*) and an electron donor $(\mathrm{Fc})$ results in positive photocurrent responses.

The wavelength of excitation $(442 \mathrm{~nm}$ ) allows the formation of the singlet $S_{2}$ state of the porphyrin, which is followed by a rapid relaxation to the singlet $S_{1}$ state. Nevertheless, the $S_{1}$ lifetime has been measured to be equal to $1.8 \mathrm{~ns}$ by timeresolved fluorescence spectroscopy, both in water and at the water|DCE interface (see Fig. S3†). ${ }^{31}$ This lifetime is too fast to envisage quenching by electron transfer from the $S_{1}$ state of the porphyrin to the Fc. Consequently, we can suppose that this $S_{1}$ state is relaxed to the triplet $T_{1}$ state, whose lifetime has been estimated to be equal to $1.3 \mathrm{~ms},{ }^{31}$ this order of magnitude allows the heterogeneous electron transfer towards the organic electron donor Fc. To justify this electron transfer from this $\mathrm{T}_{1}$ excited state of the porphyrin, we can calculate the $\Delta G$ driving force:

$$
\Delta G=F\left\{\left[E_{\mathrm{Fc}^{+} / \mathrm{Fc}}^{\mathrm{o}}\right]_{\mathrm{SHE}}^{\mathrm{o}}-\left[E_{\mathrm{ZnTPPC} / \mathrm{ZnTPPC}^{\bullet-}}^{\mathrm{o}}\right]_{\mathrm{SHE}}^{\mathrm{w}}-\Delta_{\mathrm{o}}^{\mathrm{w}} \phi\right\}-E_{\mathrm{T}_{1}}
$$

where $\left[E_{\mathrm{ZnTPPC} / \mathrm{ZnTPPC}}^{\mathrm{o}}\right]_{\mathrm{SHE}}^{\mathrm{W}}$, corresponding to the first reduction potential of the porphyrin, has been estimated to be around $-1.00 \mathrm{~V} v s$. SHE by cyclic voltammetry in aqueous media containing $\mathrm{LiCl} 0.1 \mathrm{M}$ (working electrode: mercury film electrode) (see Fig. S4 $\dagger$ ). Moreover, the oxidation potential of ferrocene, $\left[E_{\mathrm{Fc}^{+} / \mathrm{Fc}}^{\mathrm{o}}\right]_{\mathrm{SHE}}^{\mathrm{o}}$, in 1,2-dichloroethane but with respect to the aqueous standard hydrogen electrode, equals to $+0.64 \mathrm{~V} v s$. SHE. $E_{\mathrm{T}_{1}}$ corresponds to the energy of the $\mathrm{T}_{1}$ excited state of the porphyrin $(1.6 \mathrm{eV})^{31}$ and $\Delta_{\circ}^{\mathrm{w}} \phi$ is the interfacial Galvani potential difference. $F$ corresponds to the Faraday constant. These values show that the electron transfer between the excited porphyrin and ferrocene is theoretically thermodynamically favourable for $\Delta_{\circ}^{\mathrm{w}} \phi>0.04 \mathrm{~V}$, and becomes more favourable as the $\Delta_{\circ}^{\mathrm{w}} \phi$ potential difference increases.

Indeed, it can be observed that the photocurrent response increases with the applied Galvani potential difference (Fig. 3, full lines). This result is in agreement with previous published works for the heterogeneous quenching of ZnTPPC by ferrocene derivatives, ${ }^{1,3}$ and indicates that the electron transfer rate constant $\left(k_{\mathrm{et}}\right)$ increases with the applied potential in comparison to the rate constant of the excited-state decay $\left(k_{\mathrm{d}}\right)$ that is potential independent.

Moreover, whatever the applied potential, the photocurrent response always exhibits a rapid decay during illumination, followed, when the illumination is interrupted, by a negative overshoot which relaxes to zero (Fig. 3, full lines). These transient responses resemble the behavior observed for interfacial electronhole recombination at p-type semiconductor|electrolyte junctions,$^{32}$ and consequently, characterize here an important recombination rate $\left(k_{\text {rec }}\right)$ between $\mathrm{ZnTPPC}^{\bullet-}$ and $\mathrm{Fc}^{+}$ (eqn (5)). Regarding the evolution of the decays according to the applied potential, we can assume that the recombination effect is more efficient when the applied potential decreases. 
When gold NPs are added to the aqueous phase (Fig. 3, dotted line), a small increase of the generated photocurrent is observed, whatever the applied Galvani potential difference, compared to the signals recorded with a porphyrin solution without gold NPs. This enhancement of the photocurrent response can be attributed to the plasmon resonance excitation of the gold $\mathrm{NPs}$ at the interface. Indeed, excitation (at $442 \mathrm{~nm}$ ) is performed in the foot of the absorption plasmon band of the gold NPs (maximum around $520 \mathrm{~nm}$ ), allowing the excitation of the plasmon of these NPs resulting in a strong electric field locally around them. This strong electric field can further enhance the electronic excitation of porphyrins present close to the metallic NPs.

Moreover, one can also note that the rise time at low potentials is slower compared to the previous case (i.e. without gold NPs), and when the potential increases, the product separation effect becomes more efficient. Furthermore, the negative overshoot observed previously at the light-off has disappeared. These changes in the behavior of the photocurrent responses indicate the decrease of the recombination rate constant $\left(k_{\text {rec }}\right)$ in the presence of gold NPs.

Finally, in the presence of a gold film at the interface formed by a methanol addition, the photocurrent rise time is further increased before reaching a steady-state (Fig. 3, dashed lines). This behavior reveals the lack of recombination in favor of the products separation $\left(k_{\mathrm{ps}} \gg k_{\mathrm{rec}}\right)$. But the most important change is the very large enhancement of the photocurrent response compared to the two previous cases.

We have verified that the observed photocurrent response is not due to the presence of methanol used to form the gold film. Indeed, excited porphyrins are known to react with alcohol such as propan-2-ol to lead to the formation of alcohol radicals and porphyrin anion radicals. ${ }^{33}$ One can also note that this methanol added at the interface is distributed among the aqueous and the organic phase. In this way, we have performed control experiments consisting in using the biphasic system constituted of an aqueous solution of ZnTPPC without gold NPs and a DCE phase without Fc, but with methanol added at the interface. In this case, no photocurrent response has been observed. Moreover, we have also tested the system shown in Scheme 2, without gold NPs, but with methanol added at the interface. No change has been observed compared to the system without methanol (see Fig. S5†). Thus we can conclude that methanol does not react with excited porphyrins in this system, and consequently, we can ascribe the enhancement of the photocurrent response to the effect of the gold film and not to the presence of alcohol.

At this stage, it seems difficult to explain with certainty the origin of this large enhancement of the photocurrent response in the presence of the gold film. Indeed, the increase of the photocurrent response can be simply due to the increase of the quantity of gold NPs at the interface when the gold film is formed in comparison to adsorbed gold NPs from solution (without methanol, i.e. without a film).

While the plasmon resonance process observed when gold NPs are only adsorbed from the aqueous solution (no film at the interface) is undoubtedly a localized surface plasmon resonance (LSPR), in the presence of the gold film, two different plasmon resonance processes can occur. Indeed, instead of a LSPR process, a propagating surface plasmon resonance process (SPR) can take place. Briefly, when metallic nanoparticles are submitted to an electromagnetic radiation at a specific light frequency, they can interact with the conduction electrons, leading to their collective oscillation. This process is named "localized plasmon resonance". However, when a planar and well-structured metallic surface is studied instead of a colloidal solution of metallic nanoparticles, the light cannot be directly coupled with electrons, but an evanescent field, named "surface plasmon", is created that propagates along the surface. But this SPR appears only for a particular incident angle of the light onto the surface.

Consequently, in order to discriminate the plasmon resonance processes at the origin of this photocurrent enhancement in the case of the gold film, we have investigated the influence of the incident laser beam angle. Only small variations in the photocurrent response were observed, which may also be due to the variation of the cross section of the laser beam with the interface when the incident angle changes. Furthermore the photocurrent did not vary much with the light polarisation of the excitation beam, while theoretically, SPR appears only with a p-polarized laser beam.

\section{Surface plasmon resonance measurement}

Experiments to evidence a potential angle of surface plasmon resonance have also been carried out. These experiments consisted in measuring the intensity of a p-polarized laser beam after total reflection at the interface by scanning the incident angle onto the interface. The obtained SPR curve is shown in Fig. 4. A large parabola-type curve with a minimum around $80^{\circ}$ is observed. This curve is consistent with the simulated ones. As a result, even if the SPR effect occurs for the photocurrent response, it seems to be difficult to see that when the SPR effect is not very localized at a specific angle, but is extended over a large angular domain. Indeed, even if the maximum effect is observed for $80^{\circ}$, a lesser effect is also observed when one moves away from this angle.

\section{Kinetic study by IMPS}

In order to deconvolute the time constants of the various processes involved in the photoelectrochemical reaction, studies by intensity modulated photocurrent spectroscopy (IMPS) have been carried out. Theoretical considerations of this method are reported in ESI. $\dagger$

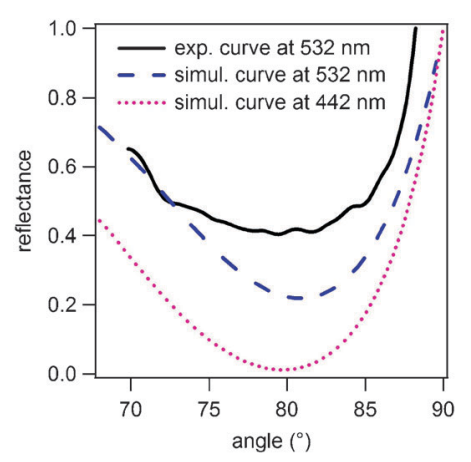

Fig. 4 Experimental SPR curve for an excitation wavelength of $532 \mathrm{~nm}$ (full line) and simulated SPR curves for excitation wavelengths of $532 \mathrm{~nm}$ (dashed line) and $442 \mathrm{~nm}$ (dotted line). 
The IMPS plots for the system with the porphyrin alone are shown in Fig. 5 for various Galvani potential differences. The graphs corresponding to the representation of the real and the imaginary components of the intensity modulated photocurrent in the complex plane (Fig. 5A) are shaped like "snails" constituting a first semicircle in the lower quadrant and a second one, which is smaller, in the upper quadrant. This shape is a typical feature for a system for which the products separation process (eqn (4)) is in great competition with the recombination process (eqn (5)), and this behavior is in agreement with the decays obtained during illumination of this system (Fig. 3, full lines). Moreover, the semicircle in the upper quadrant, which characterizes the recombination process, becomes smaller when the applied potential increases. This observation is also consistent with the fact that the higher the applied potential difference is, the more efficient the products separation is.

These IMPS data have been fitted by non-linear leastsquares routines with eqn (7) and (8) (see below and ESI $\dagger$ ), allowing a more quantitative analysis:

$$
\frac{j_{\mathrm{photo}}^{\mathrm{re}}}{g}=\frac{k_{\mathrm{ps}}\left(k_{\mathrm{rec}}+k_{\mathrm{ps}}\right)+\omega^{2}\left(1+R C_{\mathrm{int}} k_{\mathrm{rec}}\right)}{\left(1+\left(R C_{\mathrm{int}} \omega\right)^{2}\right)\left(\left(k_{\mathrm{rec}}+k_{\mathrm{ps}}\right)^{2}+\omega^{2}\right)}
$$

and

$$
\frac{j_{\text {photo }}^{\text {im }}}{g}=\frac{\omega\left(k_{\text {rec }}-R C_{\text {int }}\left(k_{\mathrm{ps}}\left(k_{\mathrm{rec}}+k_{\mathrm{ps}}\right)+\omega^{2}\right)\right)}{\left(1+\left(R C_{\mathrm{int}} \omega\right)^{2}\right)\left(\left(k_{\mathrm{rec}}+k_{\mathrm{ps}}\right)^{2}+\omega^{2}\right)}
$$

where $R C_{\text {int }}$ corresponds to the relaxation time constant of the interfacial system and $\omega$ the angular frequency of the on-off light perturbation.
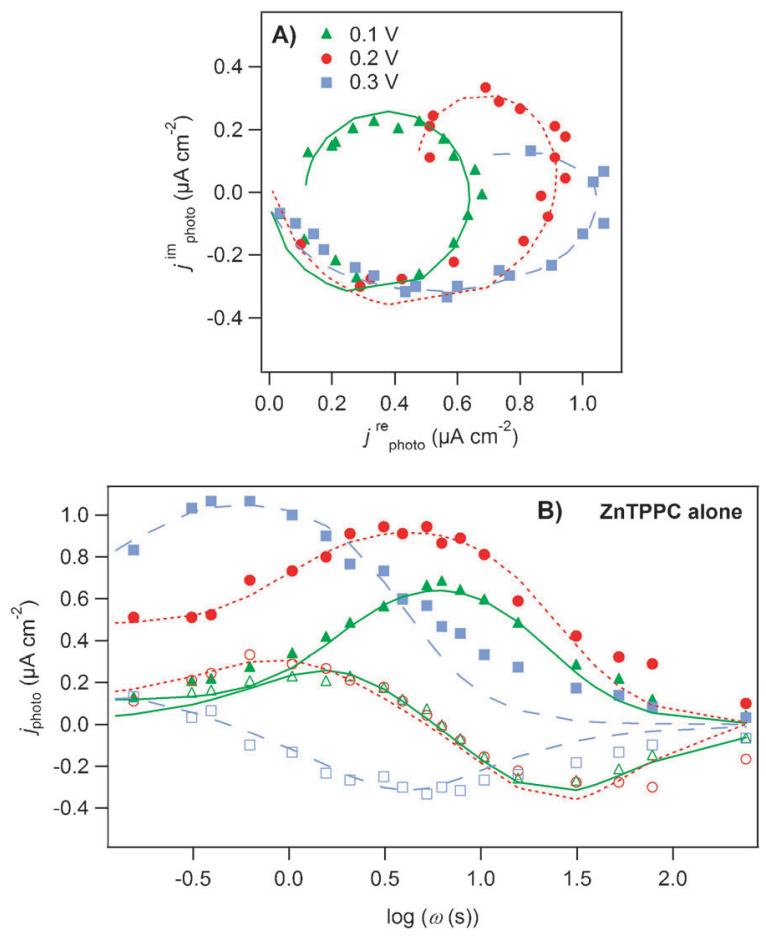

Fig. 5 (A) Complex representation of the IMPS data and (B) real and imaginary components of the intensity modulated photocurrent as a function of the angular frequency obtained from the biphasic system without gold NPs (ZnTPPC alone) at $0.1(\boldsymbol{\Delta}), 0.2(\bullet)$ and $0.3 \mathrm{~V}(\boldsymbol{\square})$.
Thus, rate constants of products separation $\left(k_{\mathrm{ps}}\right)$ and recombination $\left(k_{\text {rec }}\right)$ can be determined. Fig. 6 depicts the evolution of $k_{\mathrm{ps}}$ and $k_{\text {rec }}$, respectively, as a function of the applied Galvani potential difference. We can observe that $k_{\mathrm{ps}}$ does not change much with the applied potential whereas $k_{\text {rec }}$ decreases steeply when the applied potential increases. Consequently, in agreement with previous conclusions, the ratio $k_{\mathrm{rec}} / k_{\mathrm{ps}}$ decreases with the increase of the applied potential. Moreover, the obtained values for $k_{\mathrm{ps}}$ and $k_{\mathrm{rec}}$ are of the same order of magnitude as the ones we have obtained previously for such a biphasic system. ${ }^{4}$

The system ZnTPPC/gold NPs has been similarly studied. IMPS plots for several Galvani potential differences are shown in Fig. 7A and B. In this case, the complex representations (Fig. 7A) are more restricted in the upper part, and above $0.2 \mathrm{~V}$, only the semicircle in the lower quadrant is observable.

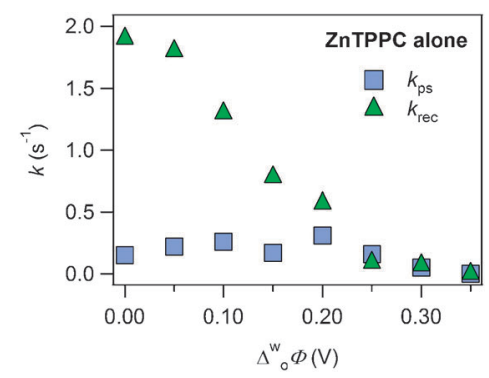

Fig. 6 Potential dependence of $k_{\mathrm{ps}}(\boldsymbol{\square})$ and $k_{\text {rec }}(\boldsymbol{\Delta})$ obtained from IMPS analysis of experiments performed in the presence of ZnTPPC alone (biphasic system without gold NPs).
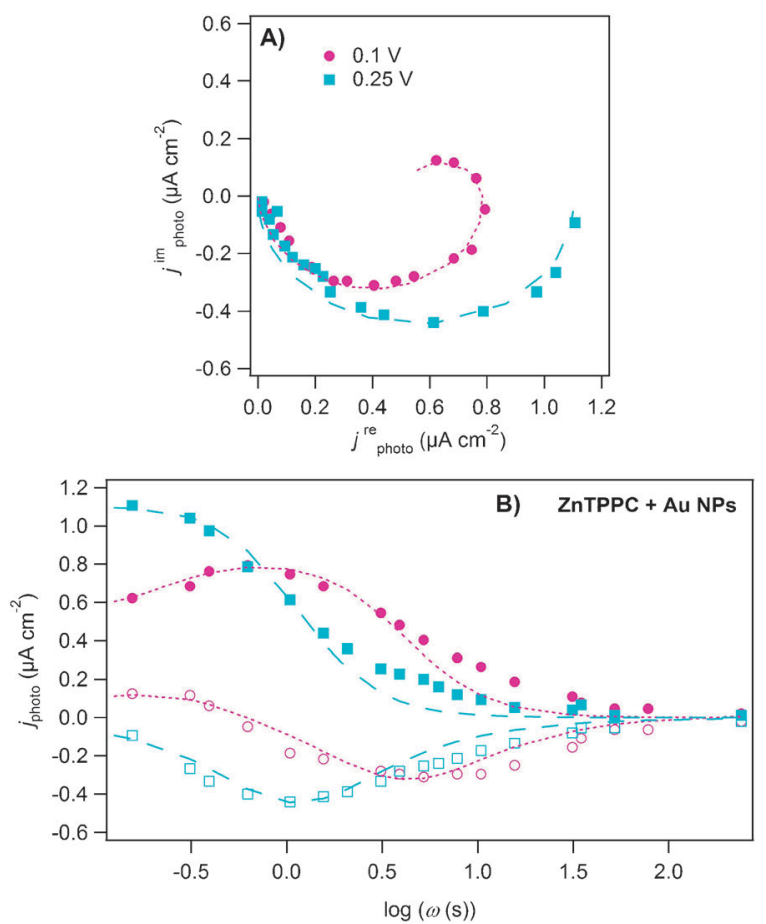

Fig. 7 (A) Complex representation of the IMPS data and (B) real and imaginary components of the intensity modulated photocurrent as a function of the angular frequency obtained from the biphasic system constituted of ZnTPPC mixed with gold NPs at $0.1(\bullet)$ and $0.25 \mathrm{~V}(\mathbf{\square})$. 


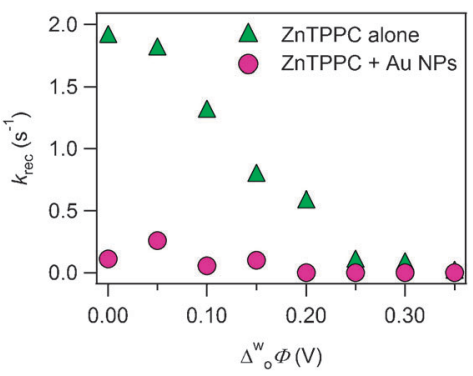

Fig. 8 Potential dependence of $k_{\text {rec }}$ obtained from IMPS analysis of experiments performed in the absence $(\boldsymbol{\Delta})$ and in the presence $(\mathbf{O})$ of gold NPs.

We can conclude that the recombination process decreases in the presence of gold NPs and above $0.2 \mathrm{~V}$, the recombination process has completely disappeared in favor of the products separation process.

Fits of these plots allow the determination of the $k_{\text {rec }}$ rate constants (Fig. 8). The $k_{\text {rec }}$ values are much lower compared to those obtained in the absence of gold NPs. Thus, the recombination process, which is very effective without gold NPs, decreases a lot when gold NPs are added to the aqueous solution of porphyrins.

Finally, similar experiments have been carried out in the presence of a gold film at the interface (Fig. 9). As expected, whatever the Galvani potential difference, the semicircles in the upper quadrant completely vanish, which is consistent with the profiles of the decays obtained during illumination of this
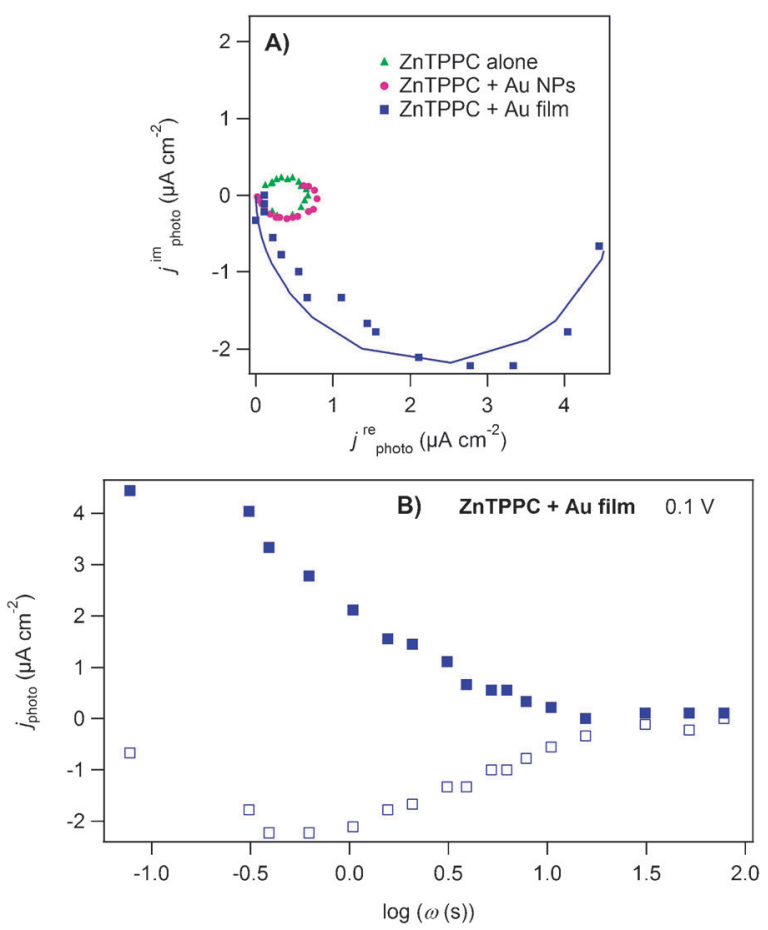

Fig. 9 (A) Complex representation of the IMPS data and (B) real and imaginary components of the intensity modulated photocurrent as a function of the angular frequency obtained at $0.1 \mathrm{~V}$ from the biphasic system constituted of ZnTPPC without gold NPs ( $\mathbf{\Delta})$, ZnTPPC mixed with gold NPs (-) and ZnTPPC in the presence of the gold film formed by addition of methanol ( $\mathbf{\square})$. system (Fig. 3, dashed lines) and confirms the absence of a recombination process in the presence of the gold film.

The major fact of this IMPS study relies on the evolution of the $k_{\text {rec }}$ rate constant. While the recombination process is very effective in the absence of gold NPs, this process significantly decreases in the presence of gold NPs (and totally disappears when the gold NP film is used). To explain this phenomenon, we can tentatively suggest that the porphyrin radical anion $\left(\mathrm{ZnTPPC}^{\bullet-}\right)$ reacts rapidly with the citrate ions surrounding the gold NPs avoiding the recombination process.

\section{Conclusions}

Effect of gold NPs and of a mirror-like gold film on the photocurrent responses associated with the heterogeneous quenching of the ZnTPPC by ferrocene has been investigated at the water|DCE interface. A very large enhancement of the photocurrent response has been observed in the presence of a gold film at the interface. This enhancement has been ascribed to a plasmon resonance process of the gold assembly, but it has not been possible to distinguish the LSPR from the SPR contribution.

A dynamic study has also been performed by IMPS experiments. Indeed, after the electron transfer (across the interface) from the excited porphyrin to ferrocene which leads to the photocurrent response, two different ways can be in competition: the recombination process (back electron transfer) and the separation of the products. IMPS studies have allowed us to show a decrease of the recombination process in the presence of gold NPs and even a complete disappearance in the presence of the gold film.

\section{Acknowledgements}

The authors are thankful to Frédéric Gumy for his technical assistance. The Swiss Science National Foundation, the EPFL and the NCCR Must project are also gratefully acknowledged for their support. One of us, HN, is grateful to EPFL for a visiting professorship.

\section{References}

1 D. J. Fermìn, Z. Ding, H. D. Duong, P. F. Brevet and H. H. Girault, Chem. Commun., 1998, 1125-1126.

2 D. J. Fermin, H. D. Duong, Z. Ding, P. F. Brevet and H. H. Girault, Electrochem. Commun., 1999, 1, 29-32.

3 D. J. Fermìn, Z. Ding, H. D. Duong, P.-F. Brevet and H. H. Girault, J. Phys. Chem. B, 1998, 102, 10334-10341.

4 D. J. Fermin, H. D. Duong, Z. Ding, P.-F. Brevet and H. H. Girault, Phys. Chem. Chem. Phys., 1999, 1, 1461-1467.

5 D. J. Fermìn, H. D. Duong, Z. Ding, P.-F. Brevet and H. H. Girault, J. Am. Chem. Soc., 1999, 121, 10203-10210.

6 H. Jensen, J. J. Kakkassery, H. Nagatani, D. J. Fermìn and H. H. Girault, J. Am. Chem. Soc., 2000, 122, 10943-10948.

7 H. A. Atwater and A. Polman, Nat. Mater., 2010, 9, 205-213.

8 B. P. Rand, P. Peumans and S. R. Forrest, J. Appl. Phys., 2004, 96, 7519-7526.

9 S.-S. Kim, S.-I. Na, J. Jo, D.-Y. Kim and Y.-C. Nah, Appl. Phys. Lett., 2008, 93, 073307.

10 A. J. Morfa, K. L. Rowlen, T. H. Reilly, M. J. Romero and J. van de Lagemaat, Appl. Phys. Lett., 2008, 92, 013504.

11 C. Hägglund, M. Zäch and B. Kasemo, Appl. Phys. Lett., 2008, 92, 013113. 
12 R. B. Konda, R. Mundle, H. Mustafa, O. Bamiduro, A. K Pradhan, U. N. Roy, Y. Cui and A. Burger, Appl. Phys. Lett., 2007, 91, 191111.

13 C. Hägglund, M. Zäch, G. Petersson and B. Kasemo, Appl. Phys. Lett., 2008, 92, 053110.

14 M. Kinkengen, J. Bergli and Y. M. Galperin, J. Appl. Phys., 2007, 102, 093713.

15 T. Akiyama, M. Nakada, N. Terasaki and S. Yamada, Chem. Commun., 2006, 395-397.

16 T. Akiyama, M. Nakada, K. Sugawa and S. Yamada, Macromol. Symp., 2008, 270, 171-176.

17 T. Akiyama, K. Aiba, K. Hoashi, M. Wang, K. Sugawa and S. Yamada, Chem. Commun., 2010, 46, 306-308.

18 I. Cohanoschi, A. Thibert, C. Toro, S. Zou and F. E. Hernàndez, Plasmonics, 2007, 2, 89-94.

19 M. Hojeij, N. Younan, L. Ribeaucourt and H. H. Girault, Nanoscale, 2010, 2, 1665-1669.

20 H. Nagatani, S. Tonari, T. Shibata and T. Sagara, Electrochem. Commun., 2011, 13, 985-988.

21 D. Yogev and S. Efrima, J. Phys. Chem., 1988, 92, 5754-5760.

22 Y.-K. Park and S. Park, Chem. Mater., 2008, 20, 2388-2393.
23 N. Younan, M. Hojeij, L. Ribeaucourt and H. H. Girault, Electrochem. Commun., 2010, 12, 912-915.

24 J. Turkevich, P. C. Stevenson and J. Hillier, Discuss. Faraday Soc., 1951, 11, 55-75

25 F. Reincke, S. G. Hickey, W. K. Kegel and D. Vanmaekelbergh, Angew. Chem., Int. Ed., 2004, 43, 458-462.

26 Y.-J. Li, W.-J. Huang and S.-G. Sun, Angew. Chem., Int. Ed., 2006, 45, 2537-2539.

27 For a list of free energies of ion transfers at various liquid|liquid interfaces, see the web site lepa.epfl.ch.

28 R. M. Corn, University of California, Irvine.

29 P. B. Johnson and R. W. Christy, Phys. Rev. B: Solid State, 1972, 6, 4370-4379.

30 F. Reincke, W. K. Kegel, H. Zhang, M. Nolte, D. Wang, D. Vanmaekelbergh and H. Möhwald, Phys. Chem. Chem. Phys., 2006, 8, 3828-3835.

31 K. Kalyanasundaram and M. Neumann-Spallart, J. Phys. Chem., 1982, 86, 5163-5169.

32 L. M. Peter, Chem. Rev., 1990, 90, 753-769.

33 D. Schaming, C. Costa-Coquelard, S. Sorgues, L. Ruhlmann and I. Lampre, Appl. Catal., A, 2010, 373, 160-167. 